\title{
Multiswitching Synchronization of a Driven Hyperchaotic Circuit Using Active Backstepping
}

\author{
A. Ayotunde Ajayi, ${ }^{1,2}$ S. Kayode Ojo, ${ }^{3}$ E. Uchechukwu Vincent, ${ }^{4,5}$ and N. Abdullahi Njah ${ }^{3}$ \\ ${ }^{1}$ Department of Physics, University of Agriculture Abeokuta, PMB 2240, Abeokuta, Nigeria \\ ${ }^{2}$ Department of Physics, Federal College of Education (Technical) Akoka, P.O. Box 269, Yaba, Lagos 101212, Nigeria \\ ${ }^{3}$ Department of Physics, University of Lagos, Akoka, Yaba, Lagos 101212, Nigeria \\ ${ }^{4}$ Department of Physical Sciences, Redeemer's University, Mowe, Ogun State, Redemption City 110115, Nigeria \\ ${ }^{5}$ Department of Physics, Lancaster University, Lancaster LA1 4YB, UK
}

Correspondence should be addressed to A. Ayotunde Ajayi; ayotundeaj@yahoo.com

Received 11 October 2013; Accepted 30 December 2013; Published 18 February 2014

Academic Editor: Dibakar Ghosh

Copyright (C) 2014 A. Ayotunde Ajayi et al. This is an open access article distributed under the Creative Commons Attribution License, which permits unrestricted use, distribution, and reproduction in any medium, provided the original work is properly cited.

\begin{abstract}
An active backstepping technique is proposed for the realization of multiswitching synchronization of periodically forced hyperchaotic Van der Pol-Duffing oscillators. The active backstepping technique is a systematic design approach with recursive procedures that skillfully optimizes the choice of Lyapunov functions and active control technique. Using the active backstepping technique, the usual master-slave synchronization scheme is extended to study the synchronization of systems with different combinations of the slave states variables with master state variables. Our numerical results confirm the effectiveness of the proposed analytical technique.
\end{abstract}

\section{Introduction}

Nonlinear deterministic dynamical systems exhibiting sensitive dependence to initial conditions (chaos) have been found to exist and not unusual. Different methods used to describe their existence are found in the fields of sciences (physical and natural), medicine, and engineering [1-3]. Various attributes of nonlinear dynamical systems such as chaos, bifurcation, multistability, pattern formation, control, and synchronization have been found useful or having potential applications in many disciplines. One of the most important attributes of nonlinear dynamical systems is that of synchronization of chaotic systems which has become more fascinating and has generated interest from researchers in recent time due to its applications in information processing, secure communication, chemical reactions, and modelling brain activity [4-7]. Increasing interest in the study of synchronization of chaotic systems has led to the discovery of various types of synchronization which include complete synchronization [8], lag synchronization [9], phase synchronization [10], generalized synchronization [11-13], measure synchronization [14-17], projective synchronization [18-20], anticipated synchronization $[21,22]$, and reduced-order synchronization [23-31]. Recently multiswitching synchronization of chaotic systems was proposed by Uçar et al. [32]. The method was successfully implemented with Lorenz system using active control technique.

Several synchronization methods have evolved to achieve stable synchronization between two or more chaotic systems. These methods include adaptive control [33], active control [34], sliding mode control [35], impulsive control [36], linear feedback control [37], backstepping control [38], open plus close loop control [39], adaptive fuzzy feedback [40], and passive control [41]. Notable among this method is the backstepping control technique which has outstanding performance in the synchronization of identical and nonidentical chaotic systems $[42,43]$. It has also been identified as a reliable design technique for stabilization and tracking [44]. In recent time, backstepping technique has been applied for controlling and 


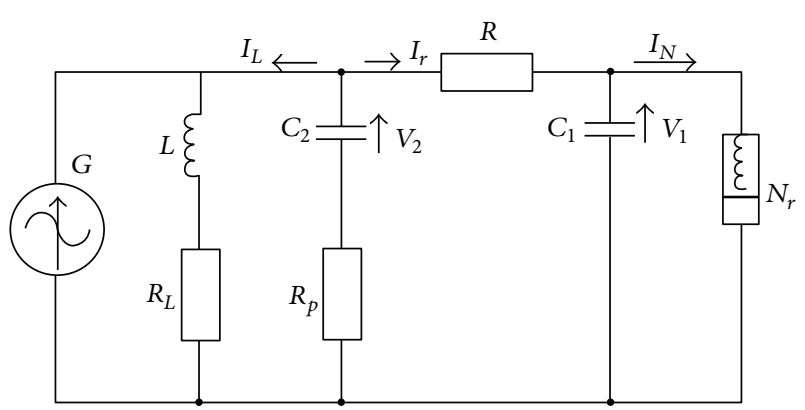

Figure 1: Unified and periodically forced Van der Pol-Duffing oscillator circuit.

tracking hyperchaotic systems [45]. In spite of the excellent performance of backstepping control technique, it has not been applied to multiswitching synchronization of chaotic system to the best of our knowledge. Motivated by the above discussion, we are reporting multiswitching synchronization of unified and periodically forced hyperchaotic Van der PolDuffing oscillator using active backstepping technique.

Van der Pol-Duffing oscillator is a popularly known and very significant classical model circuit that has been studied and even modified in some studies as reported by King et al. [46, 47], Fotsin et al. [48], and Fodjouong et al. [49]. The Van der Pol-Duffing oscillator in most reported cases is autonomous (unforced) whereas the periodically driven nonautonomous Van der Pol-Duffing oscillator considered in this paper displays more complex and richer dynamics as the amplitude and the frequency of the forcing signal are varied, thereby exhibiting chaos-hyperchaos transitions, and coexisting attractors as well as Hopf bifurcations in which quasiperiodic orbits are born [50]. Recently, there has been a renewed interest and several attempts to understand the dynamics of nonautonomous (forced) oscillators excited from equilibrium by different external forcing techniques. This is because deterministic influences often arise in practice as in cellular dynamics, blood circulation, and brain dynamics $[51,52]$.

In the present paper, we introduced the periodically forced Van der Pol-Duffing oscillator and study its synchronization. Specifically, we consider a more general form of synchronization, namely, multiswitching synchronization, in which a variable of a dynamical system synchronizes with another variable of the systems or another system. This type of synchronization was proposed by Uçar et al. [32]. Despite its relevance, it has received less attention. Here, we propose an active backstepping technique to realize the multiswitching synchronization. The rest of the paper is organized as follows: the basic dynamical properties of our model system are described in Section 2. In Section 3, the multiswitching synchronization of UVDP using active backstepping is discussed. Numerical simulations are provided in Section 4, while the paper is concluded in Section 5.

\section{Model Description}

2.1. Unified and Periodically Driven Van der Pol-Duffing Oscillator Circuit. The periodically driven Van der Pol-Duffing oscillator considered here can be modeled by the circuit shown in Figure 1, in which a dc series resistance is added to the $C_{2}$ branch of the circuit, while another dc resistance is placed parallel to it. In addition, a periodic signal generator, acting as periodic driving, is connected to the left end of the circuit as shown. By applying Kirchhoff's laws to the various branches of the circuit of Figure 1, and noting that the $i(v)$ characteristics of the nonlinear resistor $(N)$ are approximated by the cubic polynomial $i(v)=v+a v+b v^{3},(a<0, b>0)$, we obtain the following set of equations:

$$
\begin{gathered}
\frac{d V_{1}}{d t}=-\frac{1}{C_{1}}\left(\frac{1}{R}+a\right) V_{1}+\frac{V_{2}}{R C_{1}}-\frac{b}{C_{1}} V_{1}^{3}, \\
\frac{d V_{2}}{d t}=\frac{V_{1}}{R C_{2}}-\frac{\mu V_{2}}{R C_{2}}-\frac{i_{L}}{C_{2}}+\frac{i_{G}}{C_{2}} \sin \omega t, \\
\frac{d i_{L}}{d t}=\frac{V_{2}}{L}-\frac{R_{L}}{L} i_{L} .
\end{gathered}
$$

Making appropriate rescaling of (1) by setting $x=V_{1} \sqrt{b R}$, $y=V_{2} \sqrt{b R}, z=i_{L} \sqrt{b R^{3}}, \tau=t / R C_{2}, m=C_{2} / C_{1}, \alpha=-(1+$ $a R), \beta=R^{2} C_{2} / L, \gamma=R_{L} R C_{2} / L, a_{0}=R(\sqrt{b R}) I_{G}, \Omega=\omega / R C_{2}$, and $\mu=\left(R+R_{L}\right) / R_{L}$, we obtain the following dimensionless equation:

$$
\begin{gathered}
\dot{x}=-m\left(x^{3}-\alpha x-y\right), \\
\dot{y}=x-\mu y-z+a_{0} \sin \omega \tau, \\
\dot{z}=\beta y-\gamma z .
\end{gathered}
$$

System (2) is a three-dimensional nonautonomous system. By letting $w=a_{0} \sin \Omega \tau$, the system of (2) can be rewritten as a system of four-dimensional autonomous dynamical system (including $\tau$ as a dynamical variable) as follows:

$$
\begin{gathered}
\dot{x}=-m\left(x^{3}-\alpha x-y\right), \\
\dot{y}=x-\mu y-z+w, \\
\dot{z}=\beta y-\gamma z, \\
\dot{w}= \pm \omega \sqrt{a_{0}^{2}-w^{2}},
\end{gathered}
$$

where the new parameters $\omega$ and $a_{0}$ are the frequency and amplitude of the periodic driving force, respectively. Thus, our model system (2) or its equivalent system (3) has four Lyapunov exponents, allowing for hyperchaotic behaviour, that is, with the possibility of having two positive Lyapunov exponents along with one zero and one negative. Furthermore, under periodic driving, the unified Van der Pol-Duffing oscillator exhibits richer dynamical complexities, including the existence of hyperchaos-chaos transient and regime of periodic behaviour could be observed with the aid of bifurcations and Lyapunov exponents [50]. In Figure 2, we present an illustration of a typical dynamics of our model from [50]. The numerical results were performed using the standard fourth-order Runge-Kutta routine with step-size $h=2 \pi /(N \omega)$, where $N$ is the number of iterations. The 


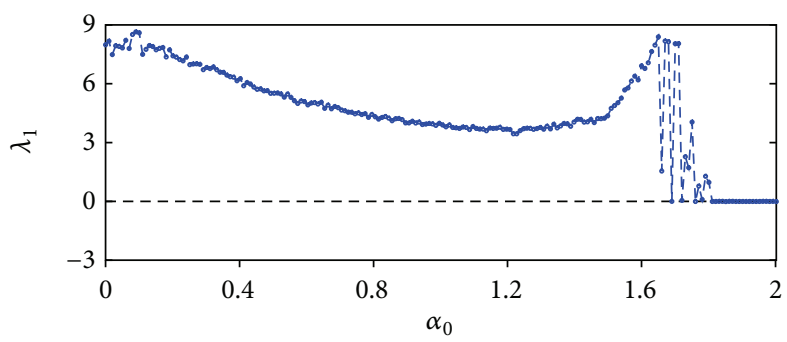

(a)

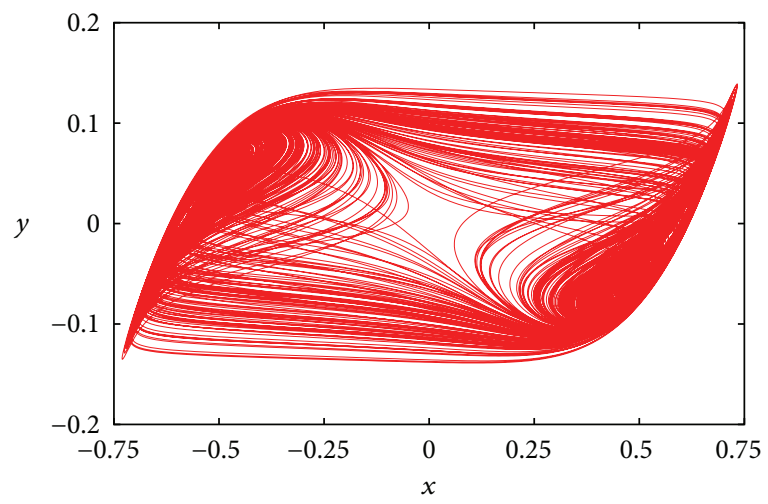

(c)

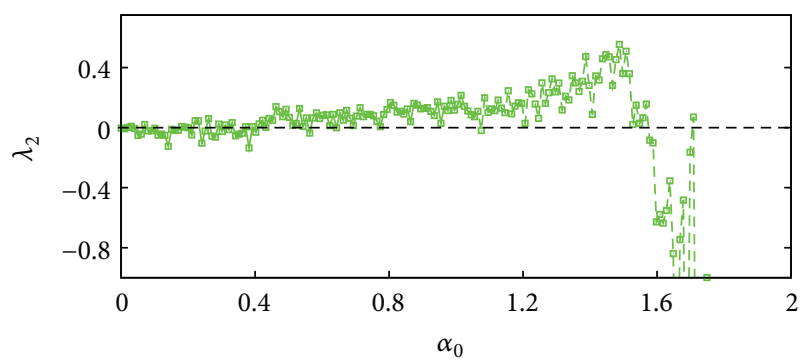

(b)

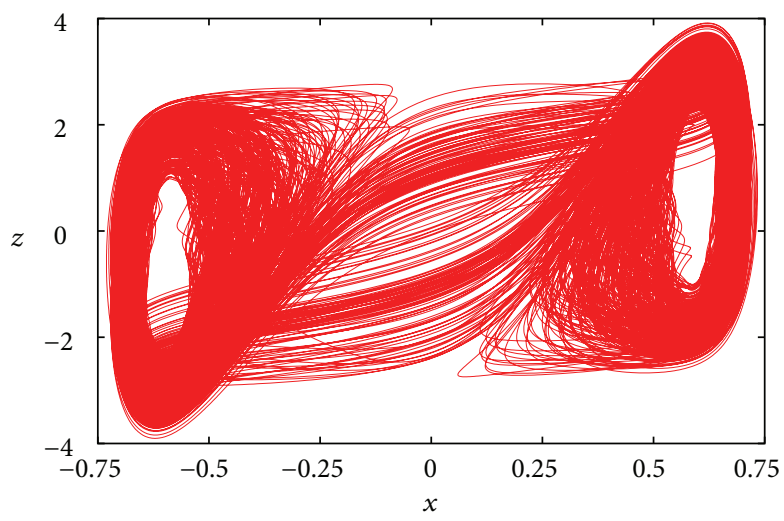

(d)

Figure 2: The first two Lyapunov exponents ( (a) $\lambda_{1}$ and (b) $\lambda_{2}$ ) as functions of the driving amplitude, $a_{0} . \alpha=0.35, \beta=300, m=100, \gamma=0.2$, $\mu=1.0$, and $\omega=10$. (c) and (d) are phase portraits of the corresponding hyperchaotic attractors for the parameters $a_{0}=1.51$ and $\omega=10$ for $y$ versus $x$ and $z$ versus $x$.

complete Lyapunov exponent spectrum was computed using the Wolf et al's algorithm [53]. For clarity and brevity, we will present only the first two exponents, namely, $\lambda_{1}$ and $\lambda_{2}$, which determine exclusively the system's behaviours. Unless otherwise stated, the following parameters were fixed: $\alpha=$ $0.35, \beta=300$, and $m=100$, while the other parameters, namely, $\mu, \gamma, a_{0}$, and $\omega$, were varied for the different cases considered. In the absence of the forcing, we obtain the Fotsin and Woafo model when $\mu=1.0$, and $\gamma=0.2$ [48], whereas, when $\mu>1.0$ and $\gamma=0$, we have the Matouk and Agiza model [54]. In Figures 2(a) and 2(b), we see clearly that $\lambda_{1}$ and $\lambda_{2}$ are positive with increasing amplitude, $a_{0}$, confirming the existence of hyperchaos in this driving amplitude regime.

2.2. Stability and Equilibria. The changes discussed above maybe understood by examining the stability of system (3). First, we obtain the fixed points by solving the general equation $F(\dot{u})=0$, where $u$ is the vector space containing $x, y$, and $z$ at $a_{0}=0$. The equilibrium points are found by equating the right-hand sides of (2) to zero such that

$$
\begin{gathered}
\dot{x}=-m\left(x^{3}-\alpha x-y\right)=0, \\
\dot{y}=x-\mu y-z=0, \\
\dot{z}=\beta y-\gamma z=0,
\end{gathered}
$$

from which the following fixed points were obtained:

$$
\begin{gathered}
E_{+}=+\left(\left(\alpha+\frac{\gamma}{\mu \gamma+\beta}\right)^{1 / 2},+\gamma \chi,+\beta \chi\right), \quad \alpha>0, \\
E_{-}=-\left(\left(\alpha+\frac{\gamma}{\mu \gamma+\beta}\right)^{1 / 2},-\gamma \chi,-\beta \chi\right), \quad \alpha<0, \\
E_{0}=(0,0,0),
\end{gathered}
$$

where

$$
\chi=\frac{(\gamma(\alpha \mu+1)+\alpha \beta)^{1 / 2}}{(\mu \gamma+\beta)^{3 / 2}} .
$$

The characteristic equation of the Jacobian matrix of system (5) about the equilibrium points $E=(\bar{x}, \bar{y}, \bar{z})$ is given as

$$
\lambda^{3}+a_{1} \lambda^{2}+a_{2} \lambda+a_{3}=0
$$

where

$$
\begin{aligned}
& a_{1}=\mu+\gamma+m\left(3 x^{2}-\alpha\right), \\
& a_{2}=m\left(3 x^{2}-\alpha\right)(\mu+\gamma)+\mu \gamma+\beta-m, \\
& a_{3}=m\left(3 x^{2}-\alpha\right)(\mu \gamma+\beta)-m \gamma .
\end{aligned}
$$




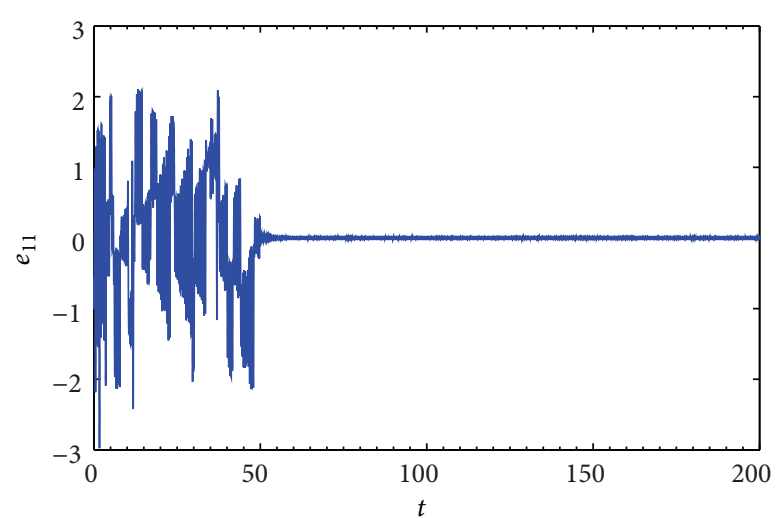

(a)

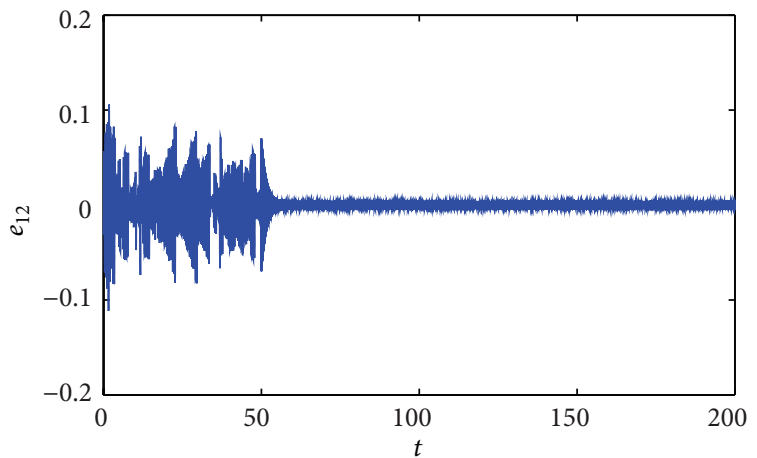

(b)

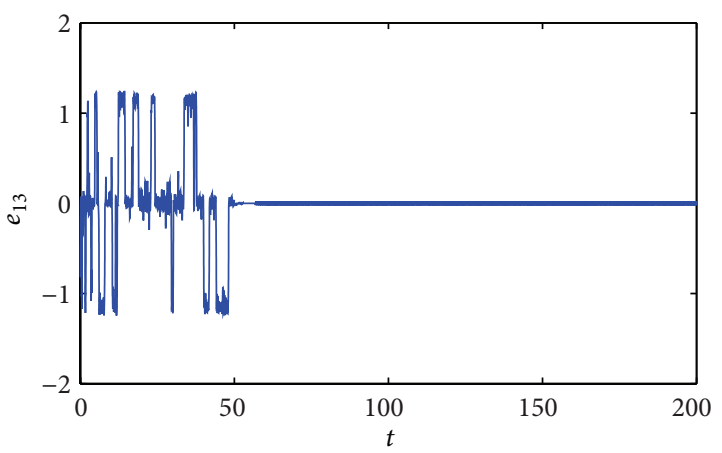

(c)

Figure 3: Error dynamics for the unified and periodically forced Van der Pol-Duffing oscillator of the system state for $N=1$ with controller defined in (24) when the control was activated at $t=50$ for (a) $e_{11}$, (b) $e_{12}$, and (c) $e_{13}$.

Before analyzing the stability properties of each points, it is important to consider the practical process of modeling system (4). It is clear that only $\alpha$ can take on negative or positive values. The other parameters are always positive. In order to study the stability conditions of the equilibrium point, $E$, we apply Routh-Hurwitz criterion [55], which states that all real eigenvalues and all real parts of complex conjugate eigenvalues are negative if and only if the following equation holds:

$$
a_{1}>0, \quad a_{3}>0, \quad a_{1} a_{2}-a_{3}>0 .
$$

At values of $m=100, \alpha=0.35, \beta=300, \gamma=0.2$, and $\mu=1.0$. For $E_{0}=(0,0,0) ; x=0, y=0, z=0$. Values of $a_{1}, a_{2}$, and $a_{3}$ obtained are $-33.8,158.2$, and -10527 , respectively.

Remark 1. The equilibrium point $E_{0}$ of the system (5) has an unstable solution when $\alpha, m, \beta, \mu$, and $\gamma$ are all positive real numbers because $a_{1}<0, a_{2}>0$, and $a_{3}<0$.

Stability Conditions of $E_{ \pm}$. When $\alpha>0$, the two equilibrium points $E_{+}$and $E_{-}$appear. The two equilibrium points are symmetric. That is, $x= \pm 0.592170771, y= \pm 0.000394518, z=$ $\pm 0.5917764 . E_{ \pm}=( \pm 0.5921707, \pm 0.0003945, \pm 0.5917764)$. Values of $a_{1}, a_{2}$, and $a_{3}$ obtained are 71.3998666, 284.4398399, and 21053.99995, respectively.
Remark $2\left(a_{1}>0, a_{2}>0, a_{3}>0\right.$, and $\left.a_{1} a_{2}>0\right)$. According to Routh-Hurwitz criteria, the equilibria $E_{+}$and $E_{-}$are stable.

\section{Multiswitching Synchronization via Backstepping}

Here, we develop multi-switching synchronization procedure based on active-backstepping and applied to the periodically forced Van der Pol-Duffing oscillator due to its richer dynamical complexities and relevance in the area of chaotic systems that can be physically built and analyzed. It is convenient to transform the driven Van der Pol-Duffing oscillator system (2) by redefining the variables as follows: $x=z, y=y$, and $z=x$ such that we have the following master system:

$$
\begin{aligned}
& \dot{x}_{1}=\beta y_{1}-\gamma x_{1}, \\
& \dot{y}_{1}=z_{1}-\mu y_{1}-x_{1}+a_{0} \sin \omega \tau, \\
& \dot{z}_{1}=-m\left(z_{1}^{3}-\alpha z_{1}-y_{1}\right)
\end{aligned}
$$

and the corresponding slave system

$$
\begin{gathered}
\dot{x}_{2}=\beta y_{2}-\gamma x_{2}+U_{11}(t), \\
\dot{y}_{2}=z_{2}-\mu y_{2}-x_{2}+a_{0} \sin \omega \tau+U_{12}(t), \\
\dot{z}_{2}=-m\left(z_{2}^{3}-\alpha z_{2}-y_{2}\right)+U_{13}(t),
\end{gathered}
$$




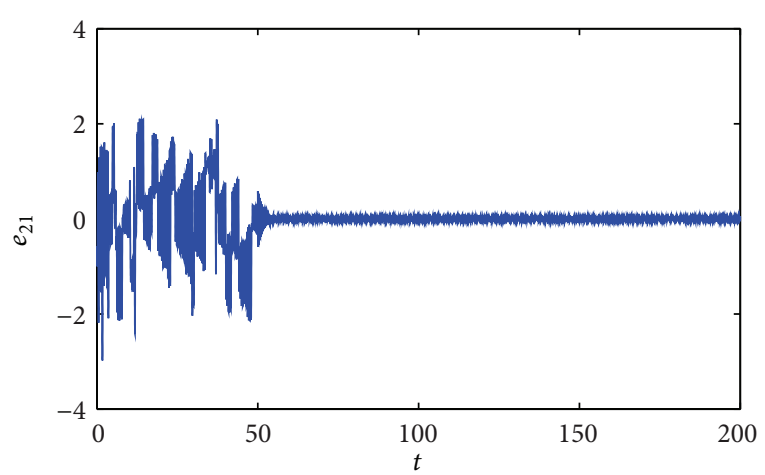

(a)

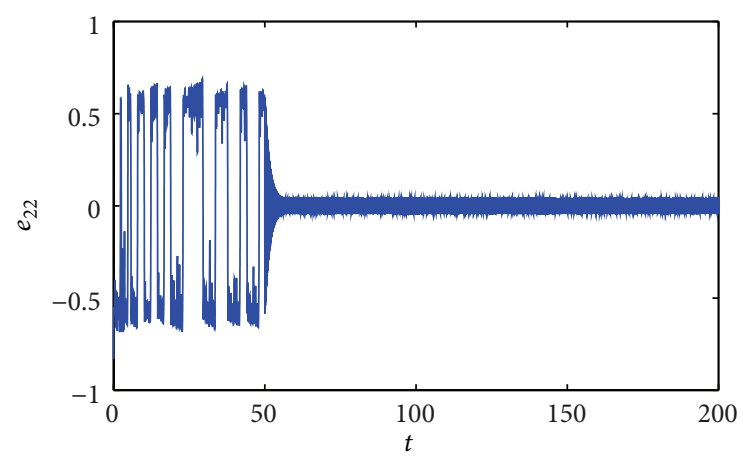

(b)

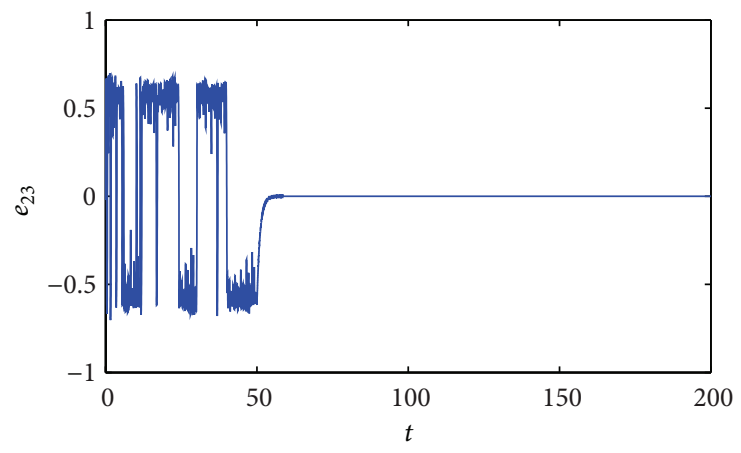

(c)

FIGURE 4: Error dynamics for the unified and periodically forced Van der Pol-Duffing oscillator of the system state for $N=2$ with controller defined in (30) when the control was activated at $t=50$ for (a) $e_{21}$, (b) $e_{22}$, and (c) $e_{23}$.

where $U_{11}(t), U_{12}(t)$, and $U_{13}(t)$ are the set of nonlinear controller for the first switch $(N=1)$. We define the error signals for this switch as $e_{11}=x_{2}-x_{1}, e_{12}=y_{2}-y_{1}$, and $e_{13}=z_{2}-z_{1}$. By considering the time derivative of the error signals together with (10) and (11), we determine the controller that would cause the three terms of error signals to asymptotically approach zero as $t \rightarrow \infty$. The controller was found from the following closed loop dynamics:

$$
\begin{aligned}
& \dot{e}_{11}=\beta e_{12}-\gamma e_{11}+U_{11}(t), \\
& \dot{e}_{12}=e_{13}-\mu e_{12}-e_{11}+U_{12}(t), \\
& \dot{e}_{13}=-m\left[z_{2}^{3}-z_{1}^{3}\right]+m \alpha e_{13}+m e_{12}+U_{13}(t) .
\end{aligned}
$$

With error dynamics represented by (12), the synchronization problem is equivalent to stabilizing the system at one of its equilibria. Since the equilibria $E_{+}$and $E_{-}$are stable and symmetric, so if appropriate $U_{11}(t), U_{12}(t)$, and $U_{13}(t)$ are chosen such that the equilibrium $E_{+}$or $E_{-}$is stable and unchanged, then asymptotic stabilization would be realized leading to globally stable synchronization of systems (10) and (11). To start with, let $q_{1}=e_{11}$, which implies that $\dot{q}_{1}=$ $\dot{e}_{11}$. Then, choose $V_{1}$ to be a Lyapunov function for the $\dot{q}_{1}$ subsystem given as

$$
V_{1}=\frac{1}{2} q_{1}^{2}
$$

and then

$$
\dot{V}_{1}=q_{1} \dot{q}_{1}=-\gamma q_{1}^{2}+q_{1}\left(\beta e_{12}+U_{11}\right) .
$$

If $e_{12}=k_{1}$ is a virtual control and a function of $e_{11}$, making $U_{11}=0$ and $k_{1}=0$, thus we obtain

$$
\dot{V}_{1}=-\gamma q_{1}^{2},
$$

which is negative definite, and this means that the subsystem, $e_{11}$, is fully stabilized. To stabilize the second subsystem in (12), let the error state between $e_{12}$ and $k_{1}$ be $q_{2}$

$$
q_{2}=e_{12}-k_{1}=e_{12}, \quad\left(k_{1}=0\right) .
$$

Introduce the second Lyapunov function $V_{2}$ given as

$$
V_{2}=V_{1}+\frac{1}{2} q_{2}^{2} .
$$

Taking time derivative of (16), we have

$$
\begin{aligned}
\dot{V}_{2}= & \dot{V}_{1}+q_{2} \dot{q}_{2}=-\gamma q_{1}^{2}-\mu q_{2}^{2} \\
& +q_{2}\left(\beta q_{1}-q_{1}+e_{13}+U_{12}\right) .
\end{aligned}
$$

Again, if $e_{13}=k_{2}=0$ is a virtual control and $U_{12}=q_{1}(1-\beta)$, then

$$
\dot{V}_{2}=-\gamma q_{1}^{2}-\mu q_{2}^{2} .
$$




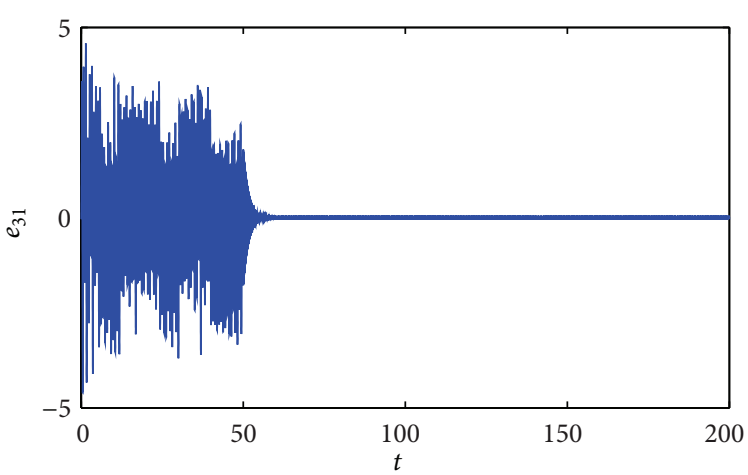

(a)

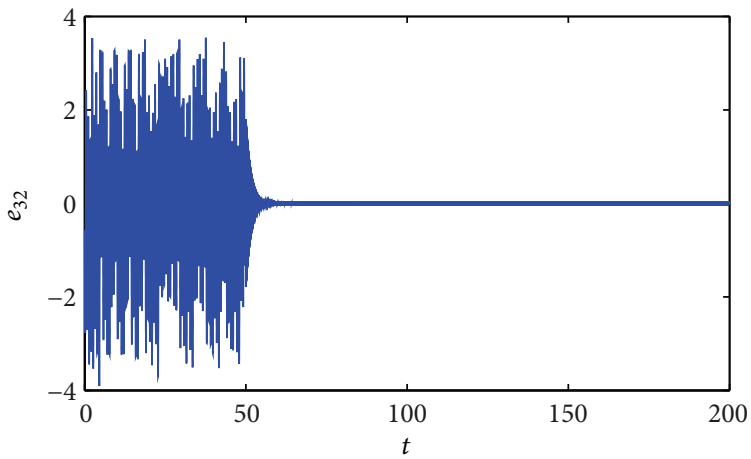

(b)

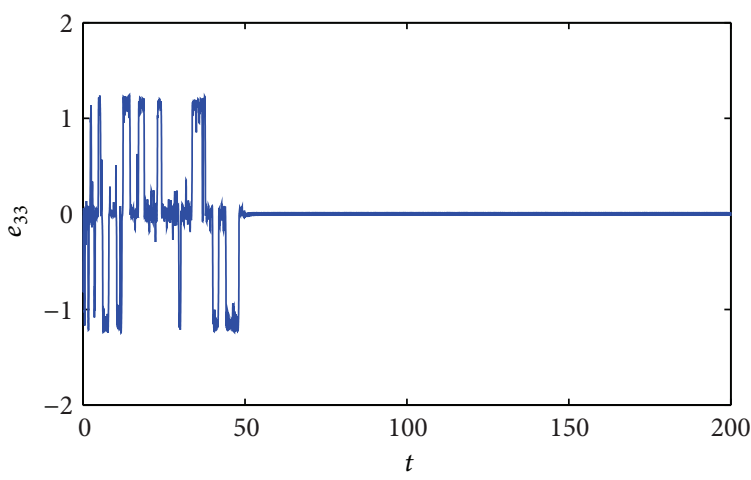

(c)

Figure 5: Error dynamics for the unified and periodically forced Van der Pol-Duffing oscillator of the system state for $N=3$ with controller defined in (31) when the control was activated at $t=50$ for (a) $e_{31}$, (b) $e_{32}$, and (c) $e_{33}$.

Equation (18) is negative definite, meaning that the subsystem, $e_{12}$, is also fully stabilized. To stabilize the third subsystem in (12), let the error state between $e_{13}$ and $k_{2}$ be $q_{3}$

$$
q_{3}=e_{13}-k_{2}=e_{13}, \quad\left(k_{2}=0\right) .
$$

We choose the third Lyapunov function and its time derivative expressed as

$$
\begin{gathered}
V_{3}=V_{2}+\frac{1}{2} q_{3}^{2}, \\
\dot{V}_{3}=\dot{V}_{2}+q_{3} \dot{q}_{3}, \\
\dot{V}_{3}=-\gamma q_{1}^{2}-\mu q_{2}^{2} \\
+q_{3}\left(q_{2}-m\left(z_{2}^{3}-z_{1}^{3}\right)+m \alpha q_{3}+m q_{2}+U_{13}(t)\right) .
\end{gathered}
$$

If the controller $U_{13}(t)$ is chosen such that

$$
U_{13}(t)=m\left(z_{2}^{3}-z_{1}^{3}\right)-q_{2}(1+m)-q_{3}(1+m \alpha),
$$

then

$$
\dot{V}_{3}=-\gamma q_{1}^{2}-\mu q_{2}^{2}-q_{3}^{2}
$$

is negative definite, so that the two systems (10) and (11) are globally asymptotically synchronized. Summarily, the virtual controllers for the system state $N=1$ are stated as follows:

$$
\begin{aligned}
U_{11} & =0, \\
U_{12} & =q_{1}(1-\beta), \\
U_{13}(t) & =m\left(z_{2}^{3}-z_{1}^{3}\right)-q_{2}(1+m)-q_{3}(1+m \alpha) .
\end{aligned}
$$

More switching states that were investigated will be presented with the parameter symbol $N=2,3,4,5$, and 6 . The error signals for these various switching states can be defined as follows:

$$
\begin{aligned}
& N=2 ; \quad e_{21}=x_{2}-x_{1} ; \quad e_{22}=y_{2}-z_{1} ; \quad e_{23}=z_{2}-y_{1}, \\
& N=3 ; \quad e_{31}=x_{2}-y_{1} ; \quad e_{32}=y_{2}-x_{1} ; \quad e_{33}=z_{2}-z_{1}, \\
& N=4 ; \quad e_{41}=x_{2}-y_{1} ; \quad e_{42}=y_{2}-z_{1} ; \quad e_{43}=z_{2}-x_{1}, \\
& N=5 ; \quad e_{51}=x_{2}-z_{1} ; \quad e_{52}=y_{2}-x_{1} ; \quad e_{53}=z_{2}-y_{1},
\end{aligned}
$$

$$
N=6 ; \quad e_{61}=x_{2}-z_{1} ; \quad e_{62}=y_{2}-y_{1} ; \quad e_{63}=z_{2}-x_{1} \text {. }
$$




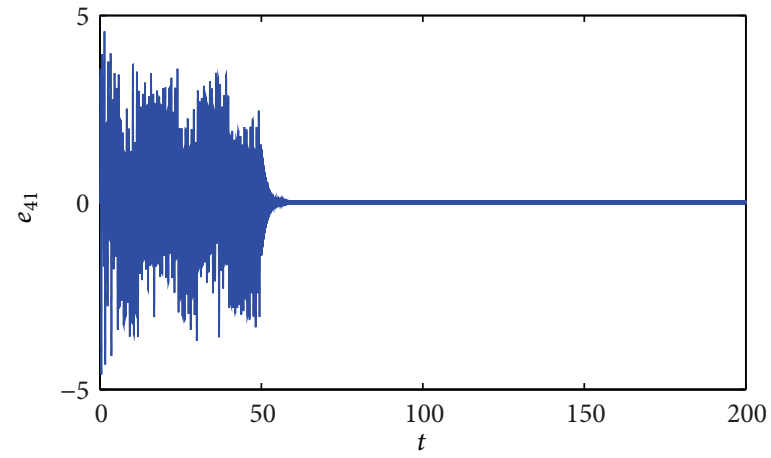

(a)

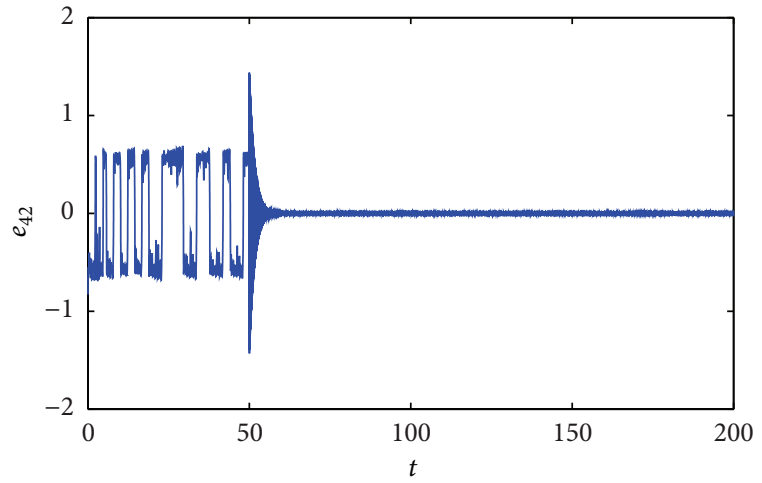

(b)

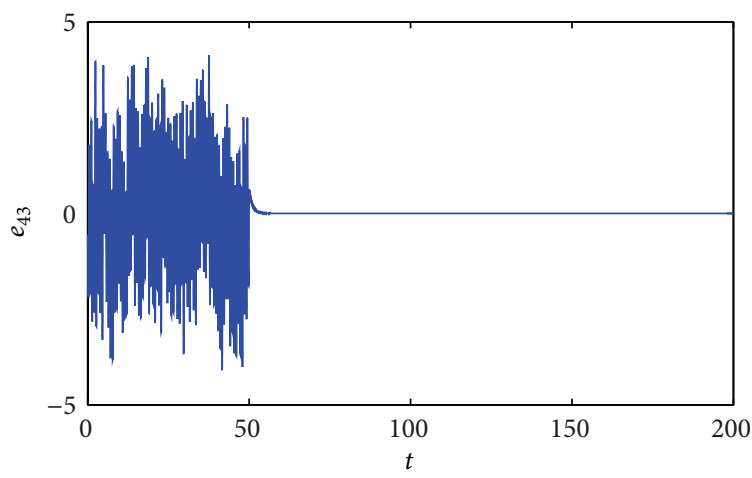

(c)

Figure 6: Error dynamics for the unified and periodically forced Van der Pol-Duffing oscillator of the system state for $N=4$ with controller defined in (32) when the control was activated at $t=50$ for (a) $e_{41}$, (b) $e_{42}$, and (c) $e_{43}$.

Following the same procedure that is itemized from (13) to (24), the controller signals $U_{N 1}(t), U_{N 2}(t)$, and $U_{N 3}(t)$ for $N=2,3,4,5$, and 6 switching, designed to cause the respective error dynamics to approach zero as time approaches infinity, are as follows:

$$
\begin{aligned}
& N=2 \Longrightarrow\left\{\begin{aligned}
U_{21}(t)= & \beta\left(y_{1}-z_{1}\right), \\
U_{22}(t)= & q_{1}(1-\beta)-y_{1}(1-m)-m z_{1}^{3} \\
& +z_{1}(m \alpha+\mu)+x_{1}-a_{0} \sin \omega t, \\
U_{23}(t)= & -q_{2}(1+m)-q_{3}(1+m \alpha) \\
& +m z_{2}^{3}-y_{1}(m \alpha+\mu)-z_{1}(m-1) \\
& -x_{1}+a_{0} \sin \omega t,
\end{aligned}\right. \\
& N=3 \Longrightarrow\left\{\begin{aligned}
U_{31}(t)= & -x_{1}(\beta+1)-y_{1}(\mu-\gamma)+z_{1} \\
& +a_{0} \sin \omega t \\
U_{32}(t)= & q_{1}(1-\beta)-x_{1}(\gamma-\mu) \\
& +y_{1}(1+\beta)-z_{1}-a_{0} \sin \omega t \\
U_{33}(t)= & -q_{3}(1+m \alpha)-q_{2}(1+m) \\
& +m\left(z_{2}^{3}-z_{1}^{3}\right)-m\left(x_{1}-y_{1}\right)
\end{aligned}\right. \\
& N=4 \Longrightarrow\left\{\begin{aligned}
U_{41}(t)= & -z_{1}(\beta-1)-y_{1}(\mu-\gamma)-x_{1} \\
& +a_{0} \sin \omega t, \\
U_{42}(t)= & q_{1}(1-\beta)-m z_{1}^{3}+z_{1}(m \alpha+\mu) \\
& +y_{1}(m+1)-x_{1}-a_{0} \sin \omega t, \\
N_{43}(t)= & -q_{3}(1+m \alpha)-q_{2}(1+m)+m z_{2}^{3} \\
& -m z_{1}+\beta y_{1}-x_{1}(m \alpha+\gamma),
\end{aligned}\right.
\end{aligned}
$$

$$
\begin{aligned}
& N=5 \Longrightarrow\left\{\begin{aligned}
& U_{51}(t)=-m z_{1}^{3}+z_{1}(m \alpha+\gamma)+m y_{1}-\beta x_{1}, \\
& U_{52}(t)=q_{1}(1-\beta)+z_{1}-y_{1}(1-\beta) \\
&-x_{1}(\gamma-\mu)-a_{0} \sin \omega t, \\
& U_{53}(t)=-q_{3}(1+m \alpha)-q_{2}(1+m)+m z_{2}^{3} \\
&+z_{1}-y_{1}(m \alpha-\mu)-x_{1}(m-1) \\
&-a_{0} \sin \omega t,
\end{aligned}\right. \\
& N=6 \Longrightarrow\left\{\begin{aligned}
& U_{61}(t)=-m z_{1}^{3}+z_{1}(m \alpha+\gamma)-y_{1}(\beta-m) \\
& U_{62}(t)=q_{1}(1-\beta)+2\left(z_{1}-x_{1}\right) \\
& U_{63}(t)=-q_{3}(1+m \alpha)-q_{2}(1+m) \\
&+m z_{2}^{3}-y_{1}(m-\beta)-x_{1}(\gamma+m \alpha) .
\end{aligned}\right.
\end{aligned}
$$

\section{Numerical Simulations}

Numerical solutions are now presented to verify the effectiveness of controllers (24) and (30)-(34). In all six cases presented, the periodically driven oscillator parameters selected remain constant at $m=100, \beta=300, \alpha=0.35, \gamma=0.2$, $\mu=1.0, a_{0}=1.51$, and $\omega=10$. This step is very important such that the hyperchaotic state obtained in [50] and shown in Figure 2 is retained and also to disable the multistability property of the system. The synchronization of the slave (13) with the master system (12) for different cases represented by $N=1,2,3,4,5$, and 6 is presented in Figures 3, 4, 5, 6, 7, and 8 . Here, we find full/complete synchronization taking place 


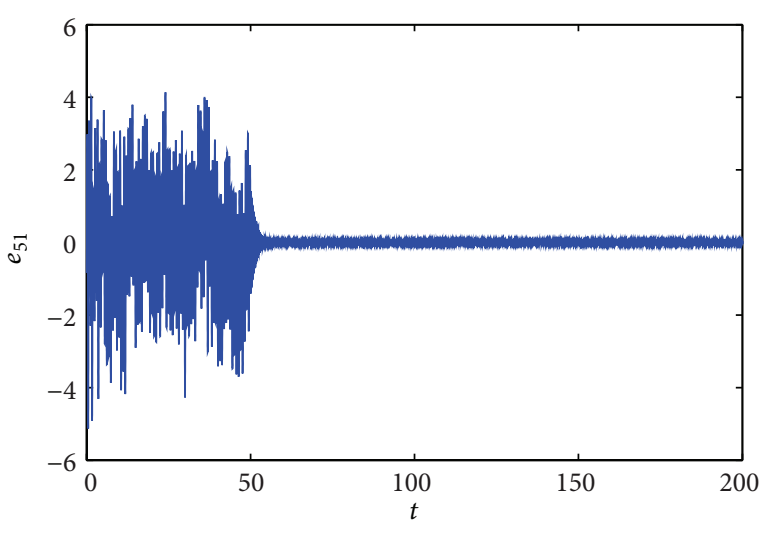

(a)

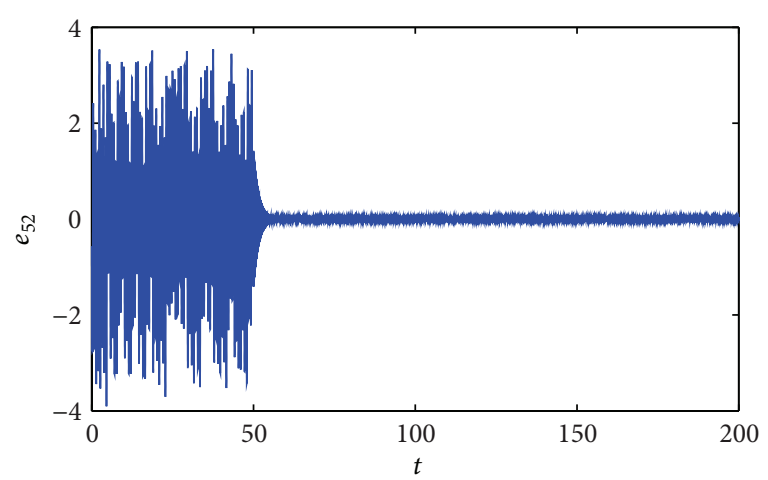

(b)

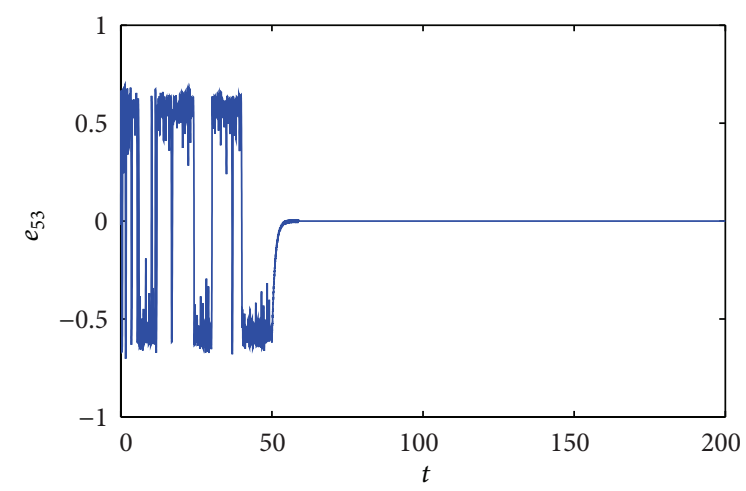

(c)

Figure 7: Error dynamics for the unified and periodically forced Van der Pol-Duffing oscillator of the system state for $N=5$ with controller defined in (33) when the control was activated at $t=50$ for (a) $e_{51}$, (b) $e_{52}$, and (c) $e_{53}$.

when each of the controllers was activated at $t \geq 50$, where the unified and periodically driven Van der Pol-Duffing oscillator was found to converge to zero as time tends to infinity which signifies that the multiswitching synchronization between systems (12) and (13) has been achieved. We remark, however, that quasi-synchronized (partial synchronization) state may also be achieved. Here, the oscillators suffer from achieving full synchronization but settle for a rather realistic form of complete synchronization wherein the state in which the

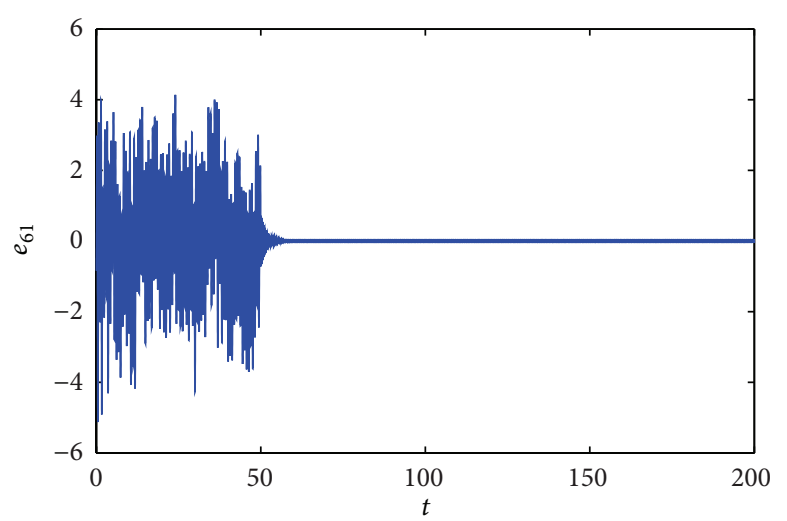

(a)

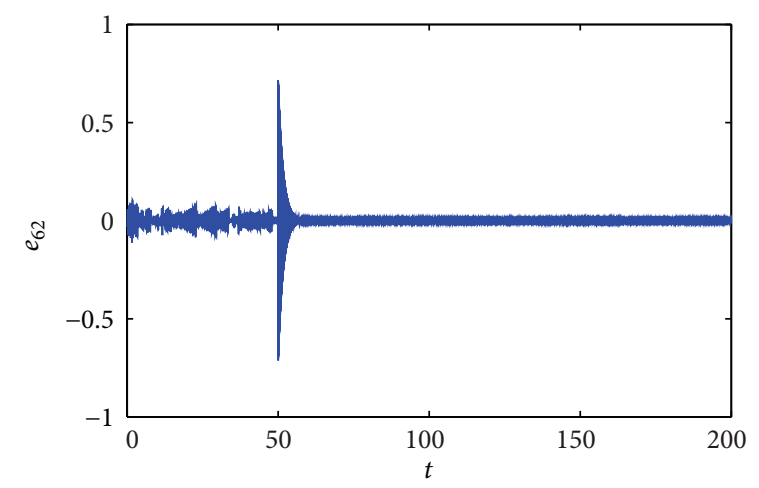

(b)

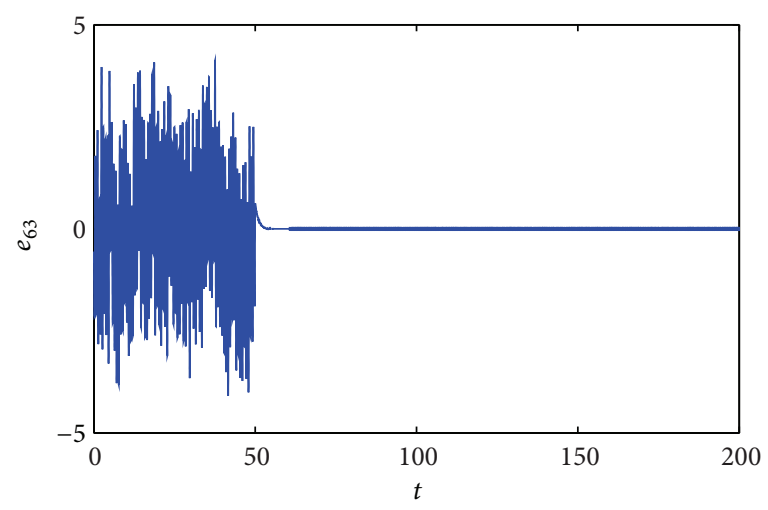

(c)

FIGURE 8: Error dynamics for the unified and periodically forced Van der Pol-Duffing oscillator of the system state for $N=6$ with controller defined in (34) when the control was activated at $t=50$ for (a) $e_{61}$, (b) $e_{62}$, and (c) $e_{63}$.

defining limit of synchronization is bounded within a definite small region around zero. That is, $\lim _{t \rightarrow \infty}\left\|e_{i j}\right\|=\epsilon \neq 0$. We find this phenomenon when for $N_{1}$, the switching is between $y_{1}$ and $y_{2}$ as well as, for $N_{3}$, when, the switching is between $y_{2}$ and $z_{2}$, denoted as $e_{12}$ and $e_{22}$ in Figures 3(b) and 4(b), respectively. This kind of synchronization phenomena are intriguing in nature as well as in practical situations and have been previously reported [56-59]. 


\section{Conclusion}

In this paper, we have presented in brief the dynamics of a unified and periodically driven Van der Pol-Duffing oscillator circuit and have shown that the model admits hyperchaotic behaviour when the amplitude of the driving force is increased with a good choice of forcing frequency. Using our model circuit, an approach for realizing multiswitching synchronization was proposed based on active backstepping technique. The numerical results obtained confirmed the effectiveness of the proposed analytical method.

\section{Conflict of Interests}

The authors declare that there is no conflict of interests regarding the publication of this paper.

\section{References}

[1] S. H. Strogatz, Nonlinear Dynamics and Chaos, Perseus Books, Reading, Mass, USA, 2000.

[2] K. T. Alligood, T. D. Sauer, and J. A. Yorke, CHAOS-an Introduction to Dynamical Systems, Springer, New York, NY, USA, 2000.

[3] R. M. Crownover, Introduction to Fractals and Chaos, Jones and Bartlett Publishers, Burlington, Mass, USA, 1995.

[4] G. Chen and X. Dong, From Chaos to Order, World Scientific, Singapore, 1998.

[5] E. Ott, C. Grebogi, and J. A. Yorke, "Controlling chaos," Physical Review Letters, vol. 64, no. 11, pp. 1196-1199, 1990.

[6] L. M. Pecora and T. L. Carroll, "Synchronization in chaotic systems," Physical Review Letters, vol. 64, no. 8, pp. 821-824, 1990.

[7] T. L. Carroll and L. M. Pecora, "Synchronizing chaotic circuits," IEEE Transactions on Circuits and Systems, vol. 38, no. 4, pp. 453-456, 1991.

[8] M. Zhan, X. Wang, X. Gong, G. W. Wei, and C.-H. Lai, "Complete synchronization and generalized synchronization of one-way coupled time-delay systems," Physical Review E, vol. 68, no. 3, Article ID 036208, 5 pages, 2003.

[9] M. G. Rosenblum, A. S. Pikovsky, and J. Kurths, "Phase synchronization of chaotic oscillators," Physical Review Letters, vol. 76, no. 11, pp. 1804-1807, 1996.

[10] M. G. Rosenblum, A. S. Pikovsky, and J. Kurths, "From phase to lag synchronization in coupled chaotic oscillators," Physical Review Letters, vol. 78, no. 22, pp. 4193-4196, 1997.

[11] V. S. Afraimovich, N. N. Verichev, and M. I. Rabinovich, "Stochastic synchronization of oscillation in dissipative systems," Radiophysics and Quantum Electronics, vol. 29, no. 9, pp. 795-803, 1986.

[12] N. F. Rulkov, M. M. Sushchik, L. S. Tsimring, and H. D. I. Abarbanel, "Generalized synchronization of chaos in directionally coupled chaotic systems," Physical Review E, vol. 51, no. 2, pp. 980-994, 1995.

[13] H. Fujiska and T. Yamada, "Stability theory of synchronized motion in coupled oscillators systems," Progress of Theoretical Physics, vol. 69, pp. 32-40, 1983.

[14] X. Wang, Z. Ying, and G. Hu, "Controlling Hamiltonian systems by using measure synchronization," Physics Letters A, vol. 298, no. 5-6, pp. 383-387, 2002.
[15] U. E. Vincent, A. N. Njah, and O. Akinlade, "Measure synchronization in a coupled Hamiltonian system associated with Nonlinear Schrödinger Equation," Modern Physics Letters B, vol. 19, no. 15, pp. 737-742, 2005.

[16] X. Wang, M. Zhan, C.-H. Lai, and H. Gang, "Measure synchronization in coupled $\chi^{4}$ Hamiltonian systems," Physical Review E, vol. 67, no. 6, Article ID 066215, 8 pages, 2003.

[17] A. Hampton and D. H. Zanette, "Measure synchronization in coupled hamiltonian systems," Physical Review Letters, vol. 83, no. 11, pp. 2179-2182, 1999.

[18] M.-F. Hu and Z.-Y. Xu, "Adaptive projective synchronization of unified chaotic systems and its application to secure communication," Chinese Physics, vol. 16, no. 11, pp. 3231-3237, 2007.

[19] Z. G. Li and D. L. Xu, "Stability criterion for projective synchronization in three-dimensional chaotic systems," Physics Letters A, vol. 282, no. 3, pp. 175-179, 2001.

[20] R. Mainieri and J. Rehacek, "Projective synchronization in three-dimensional chaotic systems," Physical Review Letters, vol. 82, no. 15, pp. 3042-3045, 1999.

[21] M. Kostur, T. P. Hänggi, and J. L. Meteos, "Anticipated synchronization synchronization in coupled inertia ratchets with timedelayed feedback: a numerical study," Physical Review E, vol. 72, Article ID 036210, 6 pages, 2005.

[22] H. U. Voss, "Anticipating chaotic synchronization," Physical Review E, vol. 61, no. 5 A, pp. 5115-5119, 2000.

[23] M. Bazhenov, R. Huerta, M. I. Rabinovich, and T. Sejnowski, "Cooperative behavior of a chain of synaptically coupled chaotic neurons," Physica D, vol. 116, no. 3-4, pp. 392-400, 1998.

[24] R. Femat and G. Solís-Perales, "Synchronization of chaotic systems with different order," Physical Review E, vol. 65, no. 3, Article ID 036226, 2002.

[25] J. H. Park, "Chaos synchronization between two different chaotic dynamical systems," Chaos, Solitons and Fractals, vol. 27, no. 2, pp. 549-554, 2006.

[26] J. H. Lü and J. A. Lu, "Controlling uncertain Lü system using linear feedback," Chaos, Solitons and Fractals, vol. 17, no. 1, pp. $127-133,2003$.

[27] J. Lu, X. Wu, and J. Lü, "Adaptive feedback of a unified system," Physics Letters A, vol. 329, pp. 327-333, 2004.

[28] M. Sun, L. Tian, and J. Xu, "Time-delay feedback control of the energy resource chaotic syatem," International Journal of Nonlinear Science, vol. 2, no. 3, pp. 166-170, 2006.

[29] A. N. Njah and K. S. Ojo, "Synchronization via backstepping nonlinear control of externally excited 6 van der pol and 6 duffing oscillators," Far East Journal of Dynamical Systems, vol. 11, no. 2, pp. 143-159, 2009.

[30] L. Li and Y. Sun, "Chaos synchronization of willis aneurysm systems," Journal of Information and Computing Science, vol. 1, no. 3, pp. 173-182, 2006.

[31] A. N. Njah and U. E. Vincent, "Synchronization and antisynchronization of chaos in an extended Bonhoffer-van der Pol oscillator using active control," Journal of Sound and Vibration, vol. 319, no. 1-2, pp. 41-49, 2009.

[32] A. Uçar, K. E. Lonngren, and E.-W. Bai, "Multi-switching synchronization of chaotic systems with active controllers," Chaos, Solitons and Fractals, vol. 38, no. 1, pp. 254-262, 2008.

[33] W. Shu-Guo and Z. Song, "Adaptive function projective synchronization of uncertain complex dynamical networks with disturbance," Chinese Physics B, vol. 22, no. 7, Article ID 070503, 2013. 
[34] S. O. Kareem, K. S. Ojo, and A. N. Njah, "Function projective synchronization of identical and non-identical modified finance and shimizu-morioka systems," Pramana Journal of Physics, vol. 79, no. 1, pp. 71-79, 2012.

[35] C.-C. Yang, "Robust synchronization and anti-synchronization of identical $\Phi 6$ oscillators via adaptive sliding mode control," Journal of Sound and Vibration, vol. 331, no. 3, pp. 501-509, 2012.

[36] J. Lu, D. W. Ho, J. Cao, and J. Kurths, "Single impulsive controller for globally exponential synchronization of dynamical networks," Nonlinear Analysis: Real World Applications, vol. 14, pp. 581-593, 2013.

[37] V. Singh, "A novel LMI-based criterion for the stability of direct-form digial filters utilizing a single two's complement nonlinearity," Nonlinear Analysis: Real World Applications, vol. 14, pp. 684-689, 2013.

[38] K. S. Ojo, A. N. Njah, and S. T. Ogunjo, "Comparison of backstepping and modified active control in projective synchronization of chaos in an extended bonhoffer-van der pol oscillator," Pramana Journal of Physics, vol. 80, no. 5, pp. 825835, 2013

[39] P. K. Roy, C. Hens, I. Grosu, and S. K. Dana, "Engineering generalized synchronization in chaotic oscillators," Chaos, vol. 21, no. 1, Article ID 013106, 2011.

[40] Y. Li, S. Tong, and T. Li, "Adaptive fuzzy output feedback control for a single-link flexible robot manipulator driven dc motor via backstepping," Nonlinear Analysis: Real World Applications, vol. 14, pp. 483-494, 2013.

[41] P. Lu, Q. Wu, and Y. Yang, "Controlling transport and synchronization in non-identical inertial ratchets," Journal of Optimization Theory and Applications, vol. 157, pp. 888-899, 2013.

[42] E.-W. Bai and K. E. Lonngren, "Synchronization of two Lorenz systems using active control," Chaos, Solitons and Fractals, vol. 8, no. 1, pp. 51-58, 1997.

[43] M.-C. Ho and Y.-C. Hung, "Synchronization of two different systems by using generalized active control," Physics Letters A, vol. 301, no. 5-6, pp. 424-428, 2002.

[44] M. Kristic, I. Kanellakopoulus, and P. O. Kototovic, Nonlinear and Adaptive Control Design, John Wiley, New York, NY, USA, 1995.

[45] H. Zhang, X.-K. Ma, M. Li, and J.-L. Zou, "Controlling and tracking hyperchaotic Rössler system via active backstepping design," Chaos, Solitons and Fractals, vol. 26, no. 2, pp. 353-361, 2005.

[46] G. P. King and S. T. Gaito, "Bistable chaos. I. Unfolding the cusp," Physical Review A, vol. 46, no. 6, pp. 3092-3099, 1992.

[47] M. G. M. Gomes and G. P. King, "Bistable chaos. II. Unfolding the cusp," Physical Review A, vol. 46, pp. 3100-3109, 1992.

[48] H. Fotsin, S. Bowong, and J. Daafouz, "Adaptive synchronization of two chaotic systems consisting of modified Van der PolDuffing and Chua oscillators," Chaos, Solitons and Fractals, vol. 26, no. 1, pp. 215-229, 2005.

[49] G. J. Fodjouong, H. B. Fotsin, and P. Woafo, "Synchronizing modified van der Pol-Duffing oscillators with offset terms using observer design: application to secure communications," Physica Scripta, vol. 75, no. 5, pp. 638-644, 2007.

[50] U. E. Vincent, B. R. N. Nbendjo, A. A. Ajayi, A. N. Njah, and P. V. E. McClintock, "Hyperchaos and chaotic transient ina driven van der pol-duffing oscillator circuit," Unpublished Report.

[51] K. Sun, X. Liu, C. Zhu, and J. C. Sprott, "Hyperchaos and hyperchaos control of the sinusoidally forced simplified Lorenz system," Nonlinear Dynamics, vol. 69, no. 3, pp. 1383-1391, 2012.
[52] T. Stankovski, A. Duggento, P. V. E. McClintock, and A. Stefanovska, "Inference of timeevolving coupled dynamical systems in the presence of noise," Physical Review Letters, vol. 109, Article ID 024101, 5 pages, 2012.

[53] A. Wolf, J. B. Swift, H. L. Swinney, and J. A. Vastano, "Determining Lyapunov exponents from a time series," Physica D, vol. 16, no. 3, pp. 285-317, 1985.

[54] A. E. Matouk and H. N. Agiza, "Bifurcations, chaos and synchronization in ADVP circuit with parallel resistor," Journal of Mathematical Analysis and Applications, vol. 341, no. 1, pp. 259-269, 2008.

[55] E. J. Routh, A Treatise on the Stability of a Given State of Motion: Particularly Steady Motion, Macmillan and co., 1877.

[56] T. Huang, C. Li, W. Yu, and G. Chen, "Synchronization of delayed chaotic systems with parameter mismatches by using intermittent linear state feedback," Nonlinearity, vol. 22, no. 3, pp. 569-584, 2009.

[57] C. Masoller, "Anticipation in the synchronization of chaotic semiconductor lasers with optical feedback," Physical Review Letters, vol. 86, no. 13, pp. 2782-2785, 2001.

[58] A. Jalnine and S.-Y. Kim, "Characterization of the parametermismatching effect on the loss of chaos synchronization," Physical Review E, vol. 65, no. 2, Article ID 026210, 7 pages, 2002.

[59] C. Li, G. Chen, X. Liao, and Z. Fan, "Chaos quasisynchronization induced by impulses with parameter mismatches," Chaos, vol. 16, no. 2, Article ID 023102, 2006. 

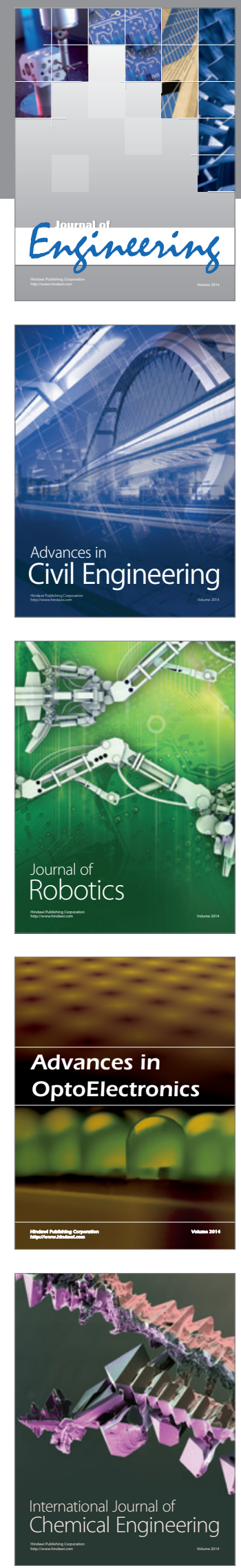

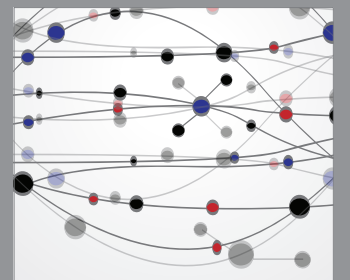

The Scientific World Journal
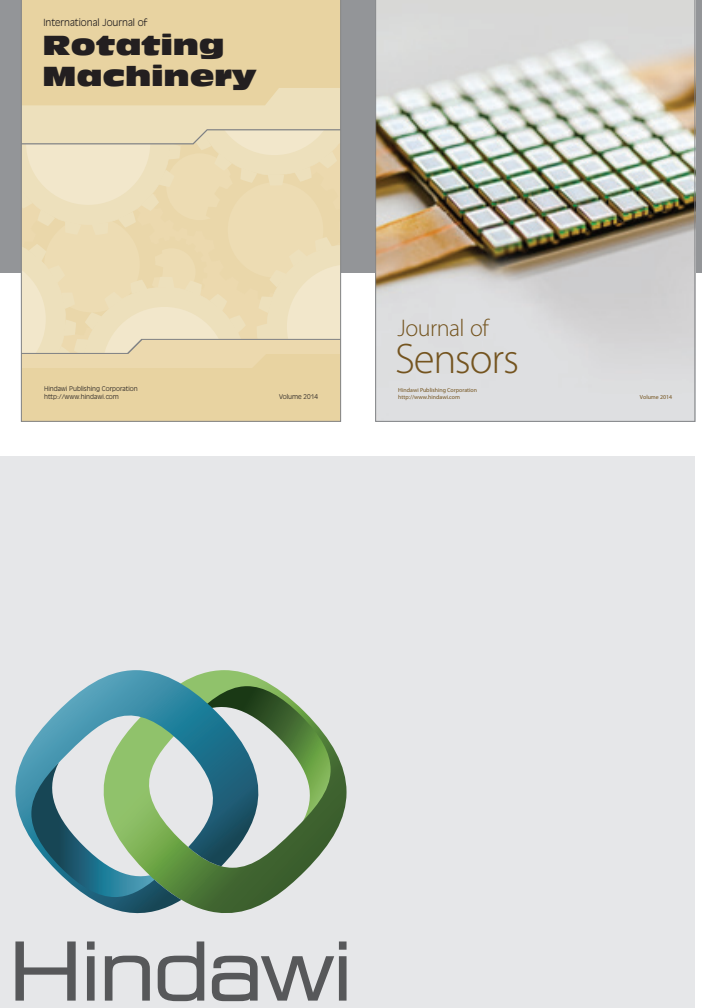

Submit your manuscripts at http://www.hindawi.com
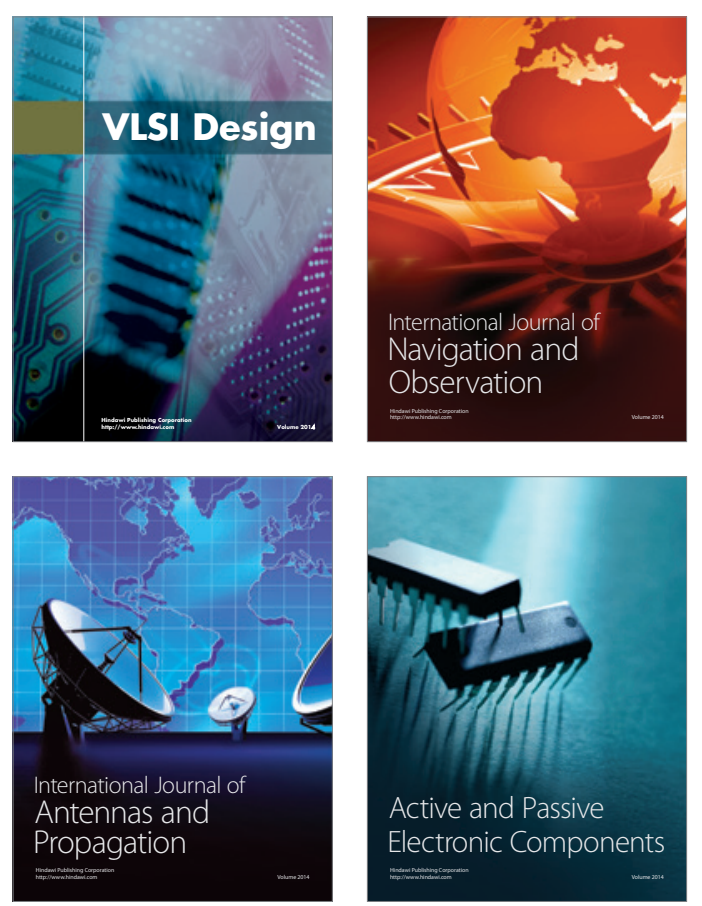
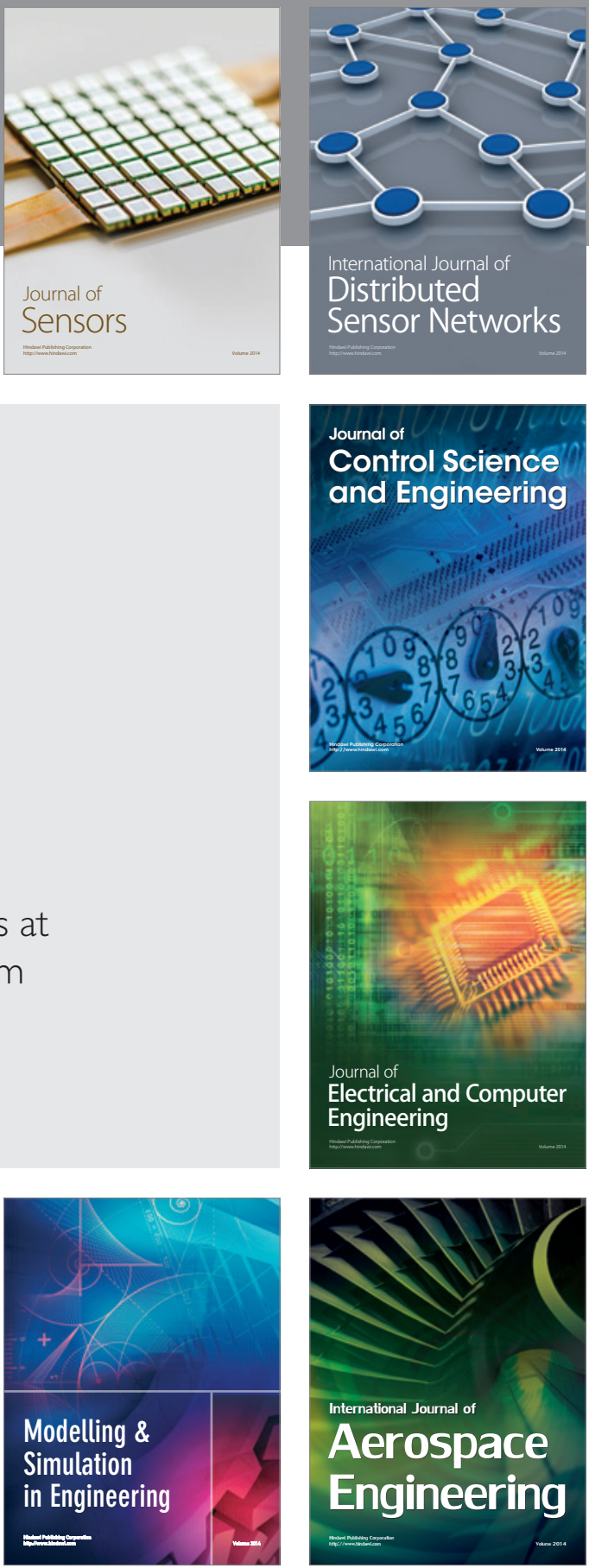

Journal of

Control Science

and Engineering
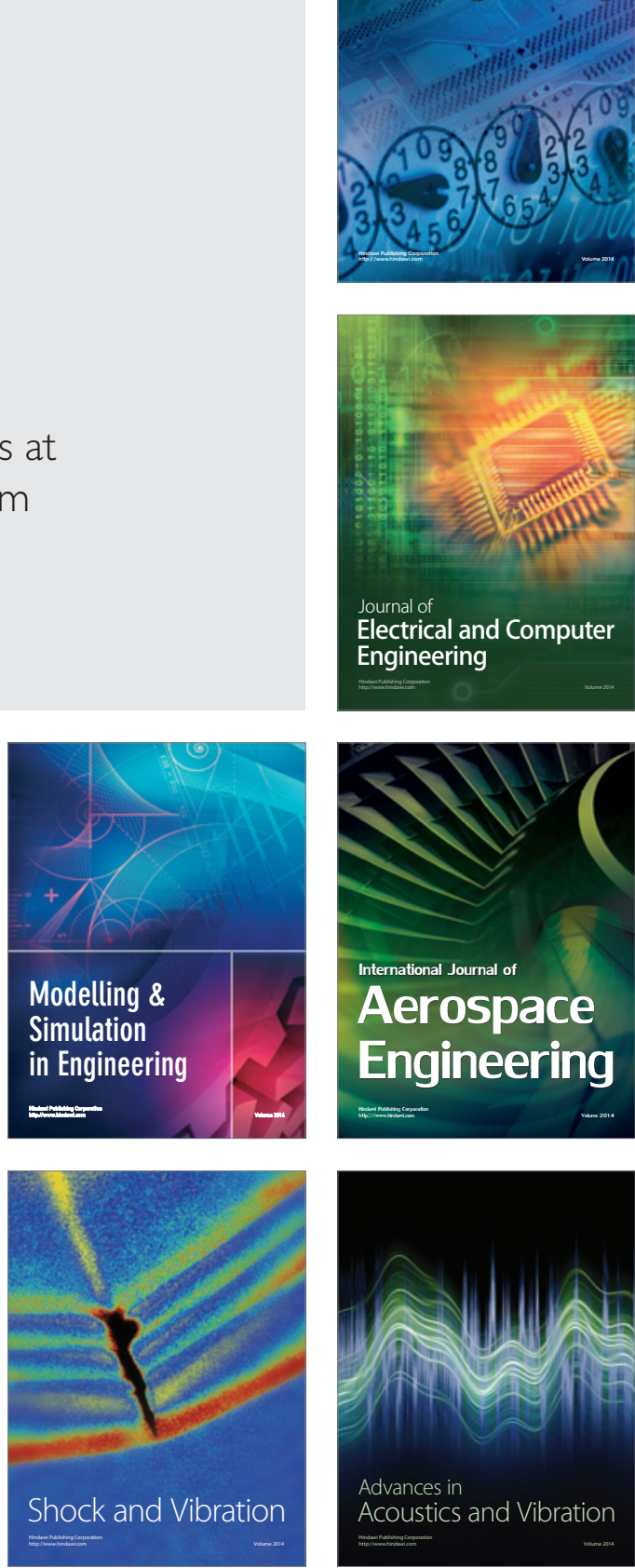